\title{
TOWARDS STRONG-COUPLING GENERALIZATION OF THE BOGOLIUBOV MODEL
}

\author{
A. Yu. Cherny ${ }^{1}$, A. A. Shanenko ${ }^{2}$ \\ ${ }^{1}$ Frank Laboratory of Neutron Physics, Joint Institute for Nuclear Research, \\ 141980, Dubna, Moscow region, Russia \\ ${ }^{2}$ Bogoliubov Laboratory of Theoretical Physics, Joint Institute for Nuclear Research, \\ 141980, Dubna, Moscow region, Russia \\ (Received April 27, 1999)
}

\begin{abstract}
The well-known results concerning a dilute Bose gas with the short-range repulsive interaction should be reconsidered due to a thermodynamic inconsistency of the method as basic to much of the present understanding of this subject and nonrelevant behaviour of the pair distribution function at small boson separations. The aim of our paper is to propose a new way of treating the dilute Bose gas with an arbitrary strong interaction. Using the reduced density matrix of the second order and a variational procedure, this way allows us to escape the inconsistency mentioned and operate with singular potentials like the Lennard-Jones one. All the consideration concerns the zero temperature.

Key words:Bose-Einstein condensation, strong-coupling regime, spatial particle correlations.
\end{abstract}

PACS numbers: 05.30.Jp, 05.30.-d, 03.75.Fi

\section{INTRODUCTION AND BASIC EQUATIONS}

It is well-known that to investigate a dilute Bose gas of particles with an arbitrary strong repulsion (the strong-coupling regime), one should go beyond the Bogoliubov approach [1] (the weak-coupling case) and treat the short-range boson correlations in a more accurate way. An ordinary manner of doing so is the use of the Bogoliubov model with the "dressed", or effective, interaction potential containing "information" on the shortrange boson correlations (see Ref. [2]). Below it is demonstrated that this manner leads to a loss of the thermodynamic consistency. To overcome this trouble, we propose a new way of investigating the strong-coupling regime which concerns the reduced density matrix of the second order (the 2 -matrix) and is based on the variational method.

The 2-matrix for the many-body system of spinless bosons can be represented as [3]:

$$
\rho_{2}\left(\mathbf{r}_{1}^{\prime}, \mathbf{r}_{2}^{\prime} ; \mathbf{r}_{1}, \mathbf{r}_{2}\right)=\frac{F_{2}\left(\mathbf{r}_{1}, \mathbf{r}_{2} ; \mathbf{r}_{1}^{\prime}, \mathbf{r}_{2}^{\prime}\right)}{N(N-1)}
$$

where the pair correlation function is given by

$$
F_{2}\left(\mathbf{r}_{1}, \mathbf{r}_{2} ; \mathbf{r}_{1}^{\prime}, \mathbf{r}_{2}^{\prime}\right)=\left\langle\psi^{\dagger}\left(\mathbf{r}_{1}\right) \psi^{\dagger}\left(\mathbf{r}_{2}\right) \psi\left(\mathbf{r}_{2}^{\prime}\right) \psi\left(\mathbf{r}_{1}^{\prime}\right)\right\rangle
$$

Here $\psi(\mathbf{r})$ and $\psi^{\dagger}(\mathbf{r})$ denote the boson field operators. Recently it has been found [4,5] that for the uniform system with a small depletion of the zero-momentum state the correlation function (2) can be written in the thermodynamic limit as follows:

$$
F_{2}\left(\mathbf{r}_{1}, \mathbf{r}_{2} ; \mathbf{r}_{1}^{\prime}, \mathbf{r}_{2}^{\prime}\right)=n_{0}^{2} \varphi^{*}(r) \varphi\left(r^{\prime}\right)
$$

$+2 n_{0} \int \frac{d^{3} q}{(2 \pi)^{3}} n_{q} \varphi_{\mathbf{q} / 2}^{*}(\mathbf{r}) \varphi_{\mathbf{q} / 2}\left(\mathbf{r}^{\prime}\right) \exp \left\{i \mathbf{q}\left(\mathbf{R}^{\prime}-\mathbf{R}\right)\right\}$

where $\mathbf{r}=\mathbf{r}_{1}-\mathbf{r}_{2}, \mathbf{R}=\left(\mathbf{r}_{1}+\mathbf{r}_{2}\right) / 2$ and similar relations take place for $\mathbf{r}^{\prime}$ and $\mathbf{R}^{\prime}$, respectively. In Eq. (3) $n_{0}=N_{0} / V$ is the density of the particles in the zeromomentum state, $n_{q}=\left\langle a_{\mathbf{q}}^{\dagger} a_{\mathbf{q}}\right\rangle$ stands for the distribution of the uncondensed bosons over momenta. Besides, $\varphi(r)$ is the wave function of a pair of particles being both condensed. In turn, $\varphi_{\mathbf{q} / 2}(\mathbf{r})$ denotes the wave function of the relative motion in a pair of bosons with the total momentum $\hbar \mathbf{q}$, this pair including one condensed and one uncondensed particle. So, Eq. (3) takes into account the condensate-condensate and supracondensatecondensate pair states and is related to the situation of a small depletion of the zero-momentum one-boson state. For the wave functions $\varphi(r)$ and $\varphi_{\mathbf{p}}(\mathbf{r})$ we have

$$
\begin{aligned}
\varphi(r) & =1+\psi(r), \\
\varphi_{\mathbf{p}}(\mathbf{r}) & =\sqrt{2} \cos (\mathbf{p r})+\psi_{\mathbf{p}}(\mathbf{r})(p \neq 0)
\end{aligned}
$$

with the boundary conditions $\psi(r) \rightarrow 0$ and $\psi_{\mathbf{p}}(\mathbf{r}) \rightarrow 0$ for $r \rightarrow \infty$. The functions $\psi(r)$ and $\psi_{\mathbf{p}}(\mathbf{r})$ can explicitly be expressed in terms of the Bose operators $a_{\mathrm{p}}^{\dagger}$ and $a_{\mathbf{p}}$ [4]:

$$
\begin{aligned}
\widetilde{\psi}(k) & =\int \psi(r) \exp (-i \mathbf{k r} \mathbf{r}) d^{3} r=\left\langle a_{\mathbf{k}} a_{-\mathbf{k}}\right\rangle / n_{0}, \\
\widetilde{\psi}_{\mathbf{p}}(\mathbf{k}) & =\int \psi_{\mathbf{p}}(\mathbf{r}) \exp (-i \mathbf{k r}) d^{3} r \\
& =\sqrt{\frac{V}{2 n_{0}}} \frac{\left\langle a_{2 \mathbf{p}}^{\dagger} a_{\mathbf{p}+\mathbf{k}} a_{\mathbf{p}-\mathbf{k}}\right\rangle}{n_{2 p}}
\end{aligned}
$$


Having in our disposal the distribution function $n_{k}$ and the set of the pair wave functions $\varphi(r)$ and $\varphi_{\mathbf{p}}(\mathbf{r})$, we are able to calculate the main thermodynamic quantities of the system of interest. In particular, the mean energy per particle is expressed in terms of $n_{k}$ and $g(r)$ via the well-known formula

$$
\varepsilon=\int \frac{d^{3} k}{(2 \pi)^{3}} T_{k} \frac{n_{k}}{n}+\frac{n}{2} \int g(r) \Phi(r) d^{3} r
$$

where $T_{k}=\hbar^{2} k^{2} / 2 m$ is the one-particle kinetic energy, $n=N / V$ stands for the boson density and the relation

$$
g(r)=F_{2}\left(\mathbf{r}_{1}, \mathbf{r}_{2} ; \mathbf{r}_{1}, \mathbf{r}_{2}\right) / n^{2}
$$

is valid for the pair distribution function $g(r)$.

\section{THE BOGOLIUBOV MODEL}

The starting point of our investigation is the weakcoupling regime which implies weak spatial correlations of particles and, thus, is characterized by the set of the inequalities

$$
|\psi(r)| \ll 1, \quad\left|\psi_{\mathbf{p}}(\mathbf{r})\right| \ll 1
$$

Specifically, the Bogoliubov model corresponds to the choice $[4,5]$

$$
|\psi(r)| \ll 1, \quad \psi_{\mathbf{p}}(\mathbf{r})=0
$$

Besides, owing to a small depletion of the Bose condensate $\left(n-n_{0}\right) / n$ we have for the one-particle density ma$\operatorname{trix} F_{1}(r)=\left\langle\psi^{\dagger}\left(\mathbf{r}_{1}\right) \psi\left(\mathbf{r}_{2}\right)\right\rangle$ :

$$
\left|\frac{F_{1}(r)}{n}\right|=\left|\int \frac{d^{3} k}{(2 \pi)^{3}} \frac{n_{k}}{n} \exp (i \mathbf{k r})\right| \leq \frac{n-n_{0}}{n} \ll 1 .
$$

So, investigating the Bose gas within the Bogoliubov scheme, we have two small quantities: $\psi(r)$ and $F_{1}(r) / n$. This enables us to write Eq. (8) with the help of (3) as follows:

$$
g(r)=1+2 \psi(r)+\frac{2}{n} \int \frac{d^{3} k}{(2 \pi)^{3}} n_{k} \exp (\mathbf{i k r})
$$

where we restricted ourselves to the terms linear in $\psi(r)$ and $F_{1}(r) / n$ and put $\psi^{*}(r)=\psi(r)$ because the pair wave functions can be chosen as real quantities. Equations for $\tilde{\psi}(k)$ and $n_{k}$ can be found varying the mean energy (7) with Eq. (11) taken into account. However, before that one should realize an important point, namely: $n_{k}$ and $\widetilde{\psi}(k)$ cannot be independent variables. Indeed, when there is no interaction between particles, there are no spatial particle correlations either. So, $\widetilde{\psi}(k)=0$ and, since the zero-temperature case is considered, all the bosons are condensed, $n_{k}=0$. While "switching on" the interaction results in appearing the spatial correlations and condensate depletion: $\tilde{\psi}(k) \neq 0$ together with $n_{k} \neq 0$. In the framework of the Bogoliubov scheme $\tilde{\psi}(k)$ is related to $n_{k}$ by the expression

$$
n_{k}\left(n_{k}+1\right)=n_{0}^{2} \widetilde{\psi}^{2}(k)
$$

Indeed, the canonical Bogoliubov transformation [1] implies that

$$
a_{\mathbf{k}}=u_{k} \alpha_{\mathbf{k}}+v_{k} \alpha_{-\mathbf{k}}^{\dagger}, \quad a_{\mathbf{k}}^{\dagger}=u_{k} \alpha_{\mathbf{k}}^{\dagger}+v_{k} \alpha_{-\mathbf{k}},
$$

where

$$
u_{k}^{2}-v_{k}^{2}=1
$$

At zero temperature $\left\langle\alpha_{\mathbf{k}}^{\dagger} \alpha_{\mathbf{k}}\right\rangle=0$ and, using Eqs. (5) and (13) we arrive at

$$
n_{k}=v_{k}^{2}, \quad \tilde{\psi}(k)=u_{k} v_{k} / n_{0}
$$

With Eqs. (14) and (15) one can readily obtain Eq. (12).

Now, let us show that all the results on the thermodynamics of a weak-coupling Bose gas can be derived for the Bogoliubov scheme with variation of the mean energy (7) under the conditions (11) and (12). Inserting Eq. (11) into Eq. (7) and, then, varying the obtained expression, we arrive at

$$
\delta \varepsilon=\int \frac{d^{3} k}{(2 \pi)^{3}}\left\{\left(T_{k}+n \widetilde{\Phi}(k)\right) \frac{\delta n_{k}}{n}+n \widetilde{\Phi}(k) \delta \tilde{\psi}(k)\right\}
$$

Relation (12) connecting $\widetilde{\psi}(k)$ with $n_{k}$ results in

$$
\delta \widetilde{\psi}(k)=\frac{\left(2 n_{k}+1\right) \delta n_{k}}{2 n_{0}^{2} \widetilde{\psi}(k)}+\frac{\widetilde{\psi}(k)}{n_{0}} \int \frac{d^{3} q}{(2 \pi)^{3}} \delta n_{q}
$$

where the equality

$$
n=n_{0}+\int \frac{d^{3} k}{(2 \pi)^{3}} n_{k}
$$

is taken into consideration. Setting $\delta \varepsilon=0$ and using Eqs. (16) and (17), we derive the following expression:

$$
\begin{aligned}
-2 T_{k} \tilde{\psi}(k) & =\frac{n^{2}}{n_{0}^{2}} \widetilde{\Phi}(k)\left(1+2 n_{k}\right) \\
& +2 n \tilde{\psi}(k)\left(\tilde{\Phi}(k)+\frac{n}{n_{0}} \int \frac{d^{3} q}{(2 \pi)^{3}} \tilde{\Phi}(q) \widetilde{\psi}(q)\right) .
\end{aligned}
$$


Here one should realize that Eq. (19) is able to yield accurate results only to the leading order in $\left(n-n_{0}\right) / n$ because the used expression for $g(r)$ given by Eq. (11) is valid to the next-to-leading order [6]. So, Eq. (19) should be rewritten as

$$
-2 T_{k} \tilde{\psi}(k)=\widetilde{\Phi}(k)\left(1+2 n_{k}\right)+2 n \tilde{\psi}(k) \Phi(k) .
$$

Equation (20) is an equation of the Bethe-Goldstone type or, in other words, the in-medium Schrödinger equation for the pair wave function. As $2 \widetilde{\Phi}(k)\left(n_{k}+\right.$ $n \widetilde{\psi}(k))$ is the product of the Fourier transforms of $\Phi(r)$ and $n(g(r)-1)$, we can rewrite Eq. (20) in a more customary form

$$
\frac{\hbar^{2}}{m} \nabla^{2} \varphi(r)=\Phi(r)+n \int \Phi(|\mathbf{r}-\mathbf{y}|)(g(y)-1) d^{3} y
$$

The structure of Eq. (21) is discussed in the papers [5,7]. Here we only remark that the right-hand side (r.h.s.) of Eq. (21) is the in-medium potential of the bosonboson interaction in the weak-coupling approximation. The system of equations (12) and (20) can easily be solved, which leads to the familiar results [1]:

$$
\begin{aligned}
& n_{k}=\frac{1}{2}\left(\frac{T_{k}+n \widetilde{\Phi}(k)}{\sqrt{T_{k}^{2}+2 n T_{k} \widetilde{\Phi}(k)}}-1\right), \\
& \tilde{\psi}(k)=-\frac{\widetilde{\Phi}(k)}{2 \sqrt{T_{k}^{2}+2 n T_{k} \tilde{\Phi}(k)}} .
\end{aligned}
$$

\section{A DILUTE BOSE GAS WiTHIN THE BOGOLIUBOV MODEL}

As already mentioned, the aim of our paper lies in investigating the case of a dilute Bose gas with an arbitrary strong repulsion between bosons. So, considering a dilute Bose gas in the weak-coupling approximation can be a good exercise providing us with useful information. Let us investigate the thermodynamics of a dilute Bose gas within the Bogoliubov model. With Eqs. (7), (11) and (22) we derive

$$
\begin{aligned}
\varepsilon & =\frac{n}{2} \widetilde{\Phi}(0) \\
& +\frac{1}{2 n} \int \frac{d^{3} k}{(2 \pi)^{3}}\left(\sqrt{T_{k}^{2}+2 n T_{k} \widetilde{\Phi}(k)}-T_{k}-n \widetilde{\Phi}(k)\right)
\end{aligned}
$$

The well-known argument of Landau (see the footnote in Ref. [1] and discussion in Ref. [2]) testifies that the properties of dilute quantum gases are ruled by the scattering length. Within the Bogoliubov model this length is usually assumed to be equal to $m \widetilde{\Phi}(0) / 4 \pi \hbar^{2}$. If so, when expanding $\varepsilon$ in powers of the boson density $n$, one could replace $\widetilde{\Phi}(k)$ by $\widetilde{\Phi}(0)$ in Eq. (23), introducing the low-momentum approximation. However, this leads to a divergency because at large $k$ the integrand behaves as $-n^{2} \widetilde{\Phi}^{2}(k) / 2 T_{k}$. To properly calculate the integral in Eq. (23), we should rewrite Eq. (23) in the following form:

$$
\varepsilon=\frac{n}{2}\left(\tilde{\Phi}(0)-\int \frac{d^{3} k}{(2 \pi)^{3}} \frac{\tilde{\Phi}^{2}(k)}{2 T_{k}}\right)+I
$$

where

$$
\begin{aligned}
I & =\frac{1}{2 n} \int_{0}^{\infty} \frac{4 \pi k^{2} d k}{(2 \pi)^{3}}\left(\sqrt{T_{k}^{2}+2 n T_{k} \widetilde{\Phi}(k)}-T_{k}\right. \\
& \left.-n \tilde{\Phi}(k)+\frac{n^{2} \widetilde{\Phi}^{2}(k)}{2 T_{k}}\right) .
\end{aligned}
$$

Now, substituting $k=(2 m n y)^{1 / 2} / \hbar$ in the integral, we obtain the expression

$$
\begin{aligned}
I & =\frac{\sqrt{2}}{4 \pi^{2}}\left(\frac{m n}{\hbar^{2}}\right)^{3 / 2} \int_{0}^{\infty} d y\{y \sqrt{y+2 \tilde{\Phi}(\sqrt{2 m n y} / \hbar)} \\
& \left.-y^{3 / 2}-\tilde{\Phi}(\sqrt{2 m n y} / \hbar) y^{1 / 2}+\frac{\tilde{\Phi}^{2}(\sqrt{2 m n y} / \hbar)}{2 \sqrt{y}}\right\}
\end{aligned}
$$

which at sufficiently small $n$ may be rewritten as

$$
\begin{aligned}
I & =\frac{\sqrt{2}}{4 \pi^{2}}\left(\frac{m n}{\hbar^{2}}\right)^{3 / 2} \int_{0}^{\infty} d y\left\{y \sqrt{y+2 \widetilde{\Phi}(0)}-y^{3 / 2}\right. \\
& \left.-\tilde{\Phi}(0) y^{1 / 2}+\frac{\widetilde{\Phi}^{2}(0)}{2 \sqrt{y}}\right\} .
\end{aligned}
$$

The derived integral is readily calculated. The result is given by

$$
I=\frac{8}{15 \pi^{2}}\left(\frac{m n}{\hbar^{2}}\right)^{3 / 2} \widetilde{\Phi}^{5 / 2}(0)
$$

In turn, the first term in the r.h.s. of Eq. (24) can be represented as

$$
\frac{n}{2}\left(\widetilde{\Phi}(0)-\int \frac{d^{3} k}{(2 \pi)^{3}} \frac{\widetilde{\Phi}^{2}(k)}{2 T_{k}}\right)=\frac{n}{2} \int \varphi^{(0)}(r) \Phi(r) d^{3} r
$$

where $\varphi^{(0)}$ is the solution of Eq. (21) in the limit $n \rightarrow 0$. This is nothing else but the Schrödinger equation in the 
Born approximation. According to relations (25) and (26) we have to conclude that the scattering length in the case of interest is expressed in the form

$$
a_{B}=\frac{m}{4 \pi \hbar^{2}} \int \varphi^{(0)}(r) \Phi(r) d^{3} r
$$

One can easily be convinced that $\widetilde{\Phi}(0)$ cannot be represented only in terms of $a_{B}$ and, hence, the dependence on the shape of the interaction potential appears in the series expansion for the mean energy in the first correction to the term $2 \pi \hbar^{2} a_{B} n / m$. To rewrite our result for $\varepsilon$ in a graphic form, we introduce one more characteristic length $b>0$ which obeys the relation

$$
b=-\frac{m}{4 \pi \hbar^{2}} \int \psi^{(0)}(r) \Phi(r) d^{3} r=\frac{m}{4 \pi \hbar^{2}} \int \frac{d^{3} k}{(2 \pi)^{3}} \frac{\Phi^{2}(k)}{2 T_{k}}
$$

where $\psi^{(0)}(r)=\varphi^{(0)}(r)-1$. Further, with the help of Eqs. (24)-(28), we arrive at

$$
\varepsilon=\frac{2 \pi \hbar^{2} a_{B} n}{m}\left\{1+\frac{128}{15 \sqrt{\pi}} \sqrt{n a_{B}^{3}}\left(1+\frac{5 b}{2 a_{B}}\right)+\cdots\right\},
$$

here the condition $b \ll a_{B}$ is of use. It is not difficult to see that the expression (28) taken with negative sign is the next correction to the scattering length calculated within the Born approximation. As for Eq. (27), it is related to the next-to-Born approximation. Note that in the Bogoliubov model the energy term $n \widetilde{\Phi}(0) / 2$ is treated as the major one [1], which implies that the condition $b \ll a_{B}$ is fulfilled. This qualitative criterion can be written as

$$
\widetilde{\Phi}(0) \gg \int \frac{d^{3} k}{(2 \pi)^{3}} \frac{\tilde{\Phi}^{2}(k)}{2 T_{k}}
$$

Beyond this inequality the model may be thermodynamically unstable. In particular, the opposite case

$$
\widetilde{\Phi}(0)<\int \frac{d^{3} k}{(2 \pi)^{3}} \frac{\widetilde{\Phi}^{2}(k)}{2 T_{k}}
$$

leads to the negative scattering length (27) which at sufficiently low densities results in $-\partial^{2} E / \partial V^{2}=\partial p / \partial V>0$.

Thus, investigated within the Bogoliubov model, the thermodynamics of a dilute Bose gas is ruled by the scattering length only in the zero-density limit. While the next-to-leading term in the series expansion given by Eq. (29) depends on the shape of the interaction, which is expressed in the appearance of the additional characteristic length $b$. This conclusion differs from the results of several papers [2] according to which the series expan- sion for $\varepsilon$ taken to the same order as that of Eq. (29), is fully determined by the scattering length. To clarify the situation concerning this difference, we should go to the strong-coupling regime.

\section{THE STRONG-COUPLING REGIME}

Now, after the detailed investigations of the Bogoliubov model within the scheme proposed, we are able to demonstrate that the investigation of the strongcoupling case based on the Bogoliubov model with the effective boson-boson interaction, results in a loss of the thermodynamic consistency. Indeed, as shown in the previous section, any calculating scheme using the basic relations of the Bogoliubov model (11), (12) conclusively leads to Eqs. (20)-(22) provided this scheme does yield the minimum of the mean energy. In this case Eqs. (20)(22) certainly includes the quantity $\Phi(r)$ which is the "bare" interaction potential appearing in Eq. (7). The use of the Bogoliubov model with the effective interaction potential substituted for $\Phi(r)$ can in no way disturb the relations given by Eqs. (11) and (12). And Eq. (7) is the same in both the weak- and strong-coupling regimes. Thus, any attempts of replacing $\Phi(r)$ by the effective "dressed" potential without modifications of Eqs. (11) and (12) results in a calculating procedure which does not really provide the minimum of the mean energy. It is nothing else but a loss of the thermodynamic consistency. We remark that we do not mean, of course, that the tmatrix approach or the pseudopotential method cannot be applied in the quantum scattering problem. It is only stated that the usual way of combining the ladder diagrams with the random phase approximation faces the trouble mentioned above. Though our present investigation is limited to the consideration of the many-boson systems, the derived result gives a hint that a similar situation is likely to take place in the Fermi case, too. In this connection it is worth noting the problem associated with the lack of self-consistency of the standard method of treating the dilute Fermi gas [8].

The strong-coupling regime is characterized by significant spatial correlations. So, Eq. (10) resulting in Eq. (11) is not relevant for an arbitrary strong repulsion between bosons at small separations when we have $\psi(0)=-1, \quad \psi_{\mathbf{p}}(0)=-\sqrt{2}$ (see Refs. [4,5]). Therefore, to investigate the strong-coupling regime, Eq. (11) should be abandoned in favour of Eq. (3). Expression (3) is accurate to the next-to-leading order in $\left(n-n_{0}\right) / n$. So, using Eqs. (3) and (8), we can write

$$
g(r)=\varphi^{2}(r)+\frac{2}{n} \int \frac{d^{3} q}{(2 \pi)^{3}} n_{q}\left(\varphi_{\mathbf{q} / 2}^{2}(\mathbf{r})-\varphi^{2}(r)\right) .
$$

Let us now perturb $\widetilde{\psi}(k)$ and $n(k)$. Working to the first order in the perturbation and keeping in mind conditions (12) and (31), from $\mathrm{Eq} .(7)$ we derive:

$$
-2 T_{k} \tilde{\psi}(k)=\tilde{U}(k)\left(1+2 n_{k}\right)+2 n \tilde{\psi}(k) \tilde{U}^{\prime}(k)
$$


with

$$
\tilde{U}(k)=\int \varphi(r) \Phi(r) \exp (-i \mathbf{k r}) d^{3} r
$$

and

$$
\widetilde{U}^{\prime}(k)=\int\left(\varphi_{\mathbf{k} / 2}^{2}(\mathbf{r})-\varphi^{2}(r)\right) \Phi(r) d^{3} r
$$

Using Eqs. (33), (34) as well as the relation $\psi_{\mathbf{k}}(\mathbf{r}) \rightarrow$ $\sqrt{2} \psi(r) \quad(k \rightarrow 0)$ (see boundary conditions (4)) [9], we obtain $\tilde{U}(0) \neq \widetilde{U}^{\prime}(0)$. This implies that the system of Eqs. (12) and (32) cannot to yield the relation $n_{k} \propto 1 / k(k \rightarrow 0)$ following from the " $1 / k^{2}$ " theorem of Bogoliubov for the zero temperature [10]. Indeed, let us assume $n_{k} \rightarrow \infty$ for $k \rightarrow 0$. Then, from Eq. (12) at $n=n_{0}$ we find $n|\widetilde{\psi}(k)| / n_{k} \rightarrow 1$ when $k \rightarrow 0$. On the contrary, Eq. (32) gives $n|\widetilde{\psi}(k)| / n_{k} \rightarrow \tilde{U}(0) / \tilde{U}^{\prime}(0) \neq 1$ for $k \rightarrow 0$. So, consideration of the Bose gas based on Eqs. (3) and (12) does not produce satisfactory results. Nevertheless, it is worth noting that Eq. (32) has an important peculiarity which differentiates it from Eq. (20) in an advantageous way. The point is that in both the limits $n \rightarrow 0$ and $k \rightarrow \infty$ Eq. (32) is reduced to

$$
-\frac{\hbar^{2}}{m} \nabla^{2} \varphi(r)+\Phi(r) \varphi(r)=0 .
$$

As one can see, this is the exact "bare" (not in-medium) Schrödinger equation, other than its Born approximation following from Eq. (21). Thus, we can expect the line of our investigation to be right.

As we have shown in the previous paragraph, an approach adequate for a dilute Bose gas with an arbitrary strong interaction can not be constructed without modifications of Eq. (12). This is also in agreement with a consequence of the relation

$$
\left|\left\langle a_{\mathrm{k}} a_{-\mathrm{k}}\right\rangle\right|^{2} \leq\left\langle a_{\mathrm{k}} a_{\mathrm{k}}^{\dagger}\right\rangle\left\langle a_{-\mathrm{k}}^{\dagger} a_{-\mathrm{k}}\right\rangle
$$

resulting from the inequality of Cauchy-SchwarzBogoliubov [10]

$$
|\langle\widehat{A} \widehat{B}\rangle|^{2} \leq\left\langle\widehat{A} \hat{A}^{\dagger}\right\rangle\left\langle\widehat{B}^{\dagger} \widehat{B}\right\rangle
$$

With Eqs. (5) and (36) one can easily derive $n_{0}^{2} \widetilde{\psi}^{2}(k) \leq$ $n_{k}\left(n_{k}+1\right)$. Thus, it is reasonable to assume that Eq. (12) takes into account only the condensate-condensate channel and ignores the supracondensate-condensate ones. Now the question arises how to find corrections to the r.h.s. of Eq. (12). At present we have no regular procedure allowing us to do this in any order of $\left(n-n_{0}\right) / n$. However, there exists an argument which makes it possible to realize the first step in this direction. The matter is that the alterations needed have to produce the equation for $\widetilde{\psi}_{\mathbf{p}}(\mathbf{k})$ which is reduced to the equation for $\widetilde{\psi}(k)$ in the limit $p \rightarrow 0$. Though this requirement does not uniquely determine the corrections to Eq. (12), it turns out to be significantly restrictive. In particular, even the simplest variant of correcting Eq. (12) in this way, leads to promising results. Indeed, this variant is specified by the expression

$$
n_{k}\left(n_{k}+1\right)=n_{0}^{2} \widetilde{\psi}^{2}(k)+2 n_{0} \int \frac{d^{3} q}{(2 \pi)^{3}} n_{q} \widetilde{\psi}_{\mathbf{q} / 2}^{2}(\mathbf{k})
$$

Eq. (37) is valid to the next-to-leading order in $(n-$ $\left.n_{0}\right) / n$. So, we may rewrite it as

$$
n_{k}\left(n_{k}+1\right)=n^{2} \widetilde{\psi}^{2}(k)+2 n \int \frac{d^{3} q}{(2 \pi)^{3}} n_{q}\left(\widetilde{\psi}_{\mathbf{q} / 2}^{2}(\mathbf{k})-\widetilde{\psi}^{2}(k)\right) .
$$

Perturbing $\widetilde{\psi}(k)$ and $n_{k}$ and bearing in mind conditions (31) and (38), Eq. (7) gives Eq. (32) again. However, now $\widetilde{U}^{\prime}(k)$ obeys the new relation

$$
\begin{aligned}
\tilde{U}^{\prime}(k) & =\int\left(\varphi_{\mathbf{k} / 2}^{2}(\mathbf{r})-\varphi^{2}(r)\right) \Phi(r) d^{3} r \\
& -\int \frac{d^{3} q}{(2 \pi)^{3}} \frac{\widetilde{U}(q)\left(\widetilde{\psi}_{\mathbf{k} / 2}^{2}(\mathbf{q})-\widetilde{\psi}^{2}(q)\right)}{\widetilde{\psi}(q)}
\end{aligned}
$$

which significantly differs from Eq. (34). Indeed, the choice of the pair wave functions as real quantities implies that operating with integrands in Eqs. (33) and (39), one can exploit $\psi_{\mathbf{p}}(\mathbf{r})-\sqrt{2} \psi(r) \propto p^{2}$ at small $p$ [11]. For $k \rightarrow 0$ this provides $\widetilde{U}^{\prime}(k)-\widetilde{U}(k)=t_{k}=c k^{4}+\cdots$. Note that for $k \rightarrow \infty$ we have $t_{k} \rightarrow-\tilde{U}(0)$. Similar to Eq. (20), Eq. (32) can yield results correct only to the leading order in $\left(n-n_{0}\right) / n$. So, it has to be solved together with Eq. (12) where $n_{0}^{2}$ should be replaced by $n^{2}$, rather than with Eq. (38). This leads to the following relations:

$$
\begin{aligned}
& n_{k}=\frac{1}{2}\left(\frac{\widetilde{T}_{k}+n \tilde{U}(k)}{\sqrt{\widetilde{T}_{k}^{2}+2 n \widetilde{T}_{k} \widetilde{U}(k)}}-1\right), \\
& \tilde{\psi}(k)=-\frac{\tilde{U}(k)}{2 \sqrt{\widetilde{T}_{k}^{2}+2 n \widetilde{T}_{k} \widetilde{U}(k)}},
\end{aligned}
$$

where $\widetilde{T}_{k}=T_{k}+n t_{k}$, with the limit $\widetilde{T}_{k} / T_{k} \rightarrow 1$ at $k \rightarrow$ $\infty$. For $k \rightarrow 0$ Eq. (40) gives $n_{k} \simeq(\sqrt{n m \widetilde{U}(0)} / \hbar k-1) / 2$, which is fully consistent with the " $1 / k^{2}$ " theorem of Bogoliubov for the zero temperature [10].

As one can see, the strong-coupling regime is more complicated than the Bogoliubov one because we do not know the quantity $\widetilde{U}(k) a b$ initio. To find it, one should solve Eqs. (33) and (41) in a self-consistent manner. 
Equations (33) and (41) lead to one more interesting relation

$$
\tilde{U}(k)=\tilde{\Phi}(k)-\frac{1}{2} \int \frac{d^{3} q}{(2 \pi)^{3}} \frac{\widetilde{\Phi}(|\mathbf{k}-\mathbf{q}|) \widetilde{U}(q)}{\sqrt{\widetilde{T}_{q}^{2}+2 n \widetilde{T}_{q} \widetilde{U}(q)}}
$$

which can be called the in-medium Lippmann-Schwinger equation for the scattering amplitude. To obtain the expansion for the energy at low densities, we must solve $\mathrm{Eq}$. (42) at $n \rightarrow 0$. Let us rewrite it in the form

$$
\tilde{U}(k)=\tilde{\Phi}(k)-\frac{1}{2} \int \frac{d^{3} q}{(2 \pi)^{3}} \frac{\tilde{\Phi}(|\mathbf{k}-\mathbf{q}|) \widetilde{U}(q)}{T_{q}}-I_{1},
$$

where for $I_{1}$ we have

$I_{1}=\frac{1}{2} \int \frac{d^{3} q}{(2 \pi)^{3}}\left\{\frac{\widetilde{\Phi}(|\mathbf{k}-\mathbf{q}|) \tilde{U}(q)}{\sqrt{\widetilde{T}_{q}^{2}+2 n \widetilde{T}_{q} \widetilde{U}(q)}}-\frac{\widetilde{\Phi}(|\mathbf{k}-\mathbf{q}|) \tilde{U}(q)}{T_{q}}\right\}$.

Operating with $I_{1}$ in the same manner as we dealt with $I$ in section III and taking into account that $t_{k}=0$ at $k=0$, for $n \rightarrow 0$ we derive

$$
I_{1}=-\alpha \tilde{\Phi}(k), \quad \alpha=\frac{\sqrt{n m^{3}}}{\pi^{2} \hbar^{3}} \tilde{U}^{3 / 2}(0)
$$

From Eqs. (42) and (43) it now follows that

$$
\begin{aligned}
\tilde{U}(k)-\widetilde{U}^{(0)}(k) & =\alpha \widetilde{\Phi}(k) \\
& -\int \frac{d^{3} q}{(2 \pi)^{3}} \frac{\tilde{\Phi}(|\mathbf{k}-\mathbf{q}|)}{2 T_{q}}\left(\tilde{U}(q)-\tilde{U}^{(0)}(q)\right) .
\end{aligned}
$$

Here $\widetilde{U}^{(0)}(k)=\int \varphi^{(0)}(r) \Phi(r) \exp (-i \mathbf{k r}) d^{3} r$ but now $\varphi^{(0)}(r)$ obeys Eq. (35) rather than Eq. (21) taken in the limit $n \rightarrow 0$ like in the section III. Let us introduce the new quantity $\widetilde{\xi}(q)=-\left(\widetilde{U}(q)-\widetilde{U}^{(0)}(q)\right) / 2 T_{q}$. Then, for its Fourier transform $\xi(r)$ we obtain

$$
-\frac{\hbar^{2}}{m} \nabla^{2}(\alpha+\xi(r))+\Phi(r)(\alpha+\xi(r))=0,
$$

here $\xi(r) \rightarrow 0$ when $r \rightarrow \infty$. Comparing Eq. (45) with Eq. (35), we find $\xi(r)=\alpha \psi^{(0)}(r)$. Hence, for $n \rightarrow 0$ we have

$$
\widetilde{U}(k) \simeq \widetilde{U}^{(0)}(k)\left(1+\gamma(k, n) \frac{8}{\sqrt{\pi}} \sqrt{n a^{3}}\right)
$$

Here $\gamma(k, n) \rightarrow 1$ when $n \rightarrow 0$. Besides, the relation $\tilde{U}^{(0)}(0)=4 \pi \hbar^{2} a / m$ is used in Eq. (46), where $a$ is the scattering length.

Having in our disposal Eq. (46), we are able to calcu- late the expansion in powers of $n$ for the condensate depletion and energy of a dilute Bose gas with an arbitrary strong interparticle potential. Considering the condensate depletion $\left(n-n_{0}\right) / n=1 /(2 \pi)^{3} \int_{0}^{+\infty} d k 4 \pi k^{2} n_{k} / n$, with the help of Eq. (40) we obtain

$$
\frac{n-n_{0}}{n}=\frac{8}{3 \sqrt{\pi}} \sqrt{n a^{3}}+\cdots
$$

Notice that according to Eq. (46) one can expect that among the omitted terms in Eq. (47) there is one proportional to $n a^{3}$.

The most simple way of deriving the expansion for the mean energy per particle is based on using the chemical potential which, in the presence of the Bose condensate, is given by

$$
\mu=\frac{1}{\sqrt{n_{0}}} \int d^{3} r^{\prime} \Phi\left(\left|\mathbf{r}-\mathbf{r}^{\prime}\right|\right)\left\langle\psi^{\dagger}\left(\mathbf{r}^{\prime}\right) \psi\left(\mathbf{r}^{\prime}\right) \psi(\mathbf{r})\right\rangle
$$

This formula follows from the well-known expression $\delta \Omega=\langle\delta(\hat{H}-\mu \hat{N})\rangle$, where $\delta \Omega$ is an infinitesimal change of the grand canonical potential, and relation (see Ref. [10])

$$
\frac{\partial \Omega\left(N_{0}, \mu, T\right)}{\partial N_{0}}=0 .
$$

Using the specific expressions for the scattering parts of the condensate-condensate and supracondensatecondensate pair wave functions [4] given by Eqs. (5) and (6) one can represent Eq. (48) in the following form:

$$
\mu=n_{0} \tilde{U}(0)+\sqrt{2} \int \frac{d^{3} q}{(2 \pi)^{3}} n_{q} \tilde{U}_{\mathbf{q} / 2}(\mathbf{q} / 2)
$$

here

$$
\widetilde{U}_{\mathbf{p}}(\mathbf{k})=\int \varphi_{\mathbf{p}}(\mathbf{r}) \Phi(r) \exp (-i \mathbf{k r}) d^{3} r .
$$

Now, for $n \rightarrow 0$ (see the procedure of calculating the integral $I$ in section II one can rewrite Eq. (49) as

$$
\mu=n \tilde{U}(0)\left(1+\frac{n-n_{0}}{n}+\cdots\right)
$$

Inserting Eqs. (46) and (47) into Eq. (50), we arrive at

$$
\mu=\frac{4 \pi \hbar^{2} a n}{m}\left(1+\frac{32}{3 \sqrt{\pi}} \sqrt{n a^{3}}+\cdots\right) .
$$

This result for the chemical potential implies, due to the basic thermodynamic formula $\mu=\partial(\varepsilon n) / \partial n$, the following expansion for the mean energy per particle:

$$
\varepsilon=\frac{2 \pi \hbar^{2} a n}{m}\left(1+\frac{128}{15 \sqrt{\pi}} \sqrt{n a^{3}}+\cdots\right) .
$$




\section{A. YU. CHERNY, A. A. SHANENKO}

Relation (52) coincides with the well-known result of the approach in [2] reduced to the Bogoliubov model with the "dressed" interaction. It is not a surprise because according to the conclusions of section II, we know that the numerical factor $128 /(15 \sqrt{\pi})$ appears in the series expansion for the mean energy per particle within the Bogoliubov model (see Eq. (29)). Replacing the bare interaction potential by the "dressed" one results in replacing the scattering length $a_{B}$ in Eq.(29) by its exact value $a$. The only problem of doing so concerns the parameter $b$. Indeed, it follows from Eq. (28) that substituting the hard-sphere potential $\tilde{U}(0)=4 \pi \hbar^{2} a / m$ for $\tilde{\Phi}(k)$ leads to the familiar divergency (see, e.g. Ref. [8], p. 314). This obstacle has been overcome with the help of the well-known argument of Landau (see the footnote in the paper [1]) stating that the thermodynamics of dilute quantum gases is only ruled by the vacuum scattering amplitude. According to this reasoning one can expect that the dependence on the shape of the interaction potential should not appear in the first orders of the density series expansion of the thermodynamic quantities. So, various regularizing procedures, more or less speculative, have been worked out in order to exclude this divergence (together with parameter $b$ ). On the contrary, there are no problems like this within the approach of the present paper. Here Eq. (52) is derived on the solid theoretical basis rather than with the help of Landau's argument. In spite of its reasonable character, it needed to be corroborated, and the results of this paper given by Eqs. (47), (50) and (52) have proved the validity of Landau's argument beyond any inconsistencies and divergencies. In the weak-coupling case when $|\Phi(r)| \ll 1$, the energy per particle calculated within our scheme is expressed by Eq. (52) with a replaced by $a_{B}$. So, the appearance of the parameter $b$ in the results of section II is an artefact following from the neglect of scattering in the supracondensate-condensate pair wave channel.

The divergence mentioned in the previous paragraph is not typical of the strong-coupling perturbation theory for the many-boson systems but results from, say, the weak-coupling spirit of the approach of Ref. [2]. A simple way to be convinced of this is to consider the spatial boson correlations. Taken to the lowest-order with respect to the density, the structural factor (see the last paper in Ref. [2]) is of the form

$$
S(k)=\frac{T_{k}}{\sqrt{T_{k}^{2}+2 n T_{k} \widetilde{U}^{(0)}(k)}} .
$$

By definition we have

$$
g(r)=1+\frac{1}{n} \int \frac{d^{3} k}{(2 \pi)^{3}}(S(k)-1) \exp (i \mathbf{k r})
$$

Using Eqs. (53) and (54), for $n \rightarrow 0$ one can readily find

$$
g(r) \rightarrow 1+2 \psi^{(0)}(r)
$$

where $\psi^{(0)}(r)$ obeys Eq. (35). This result answers approximation $(11)$ while $\psi^{(0)}(r)$ is not related to the weakcoupling regime and obeys the exact "bare" Schrödinger equation. In the situation $\Phi(r) \rightarrow \infty$ for $r \rightarrow 0$ one has $\psi^{(0)}(r=0)=-1$, which implies, according to Eq. (55), $g(r=0) \rightarrow-1$ for $n \rightarrow 0$. It is not consistent with the physical sense of $g(r)$ and has nothing to do with the strong-coupling case corresponding to Eq. (31) when for $n \rightarrow 0$

$$
g(r) \rightarrow\left(1+\psi^{(0)}(r)\right)^{2}
$$

Notice that the zero-density limits for the thermodynamic quantities of a strongly interacting dilute Bose gas were first found in the Bogoliubov original paper [1]:

$$
\left(n-n_{0}\right) / n \rightarrow 0, g(r) \rightarrow\left(\varphi^{(0)}(r)\right)^{2}, \varepsilon / n \rightarrow \widetilde{U}^{(0)}(0) / 2
$$

At last, we remark that due to the incorrect picture of the spatial boson correlations found in papers [2], one can expect significant alterations for the spectrum of the elementary excitations too. However, to clarify these corrections we should conclusively solve the problem concerning relation between the momentum distribution and scattering parts of the pair wave functions. Indeed, it has been mentioned that there exist various possibilities of generalizing Eq. (12) so as to obtain the equation for $\widetilde{\psi}_{\mathbf{p}}(\mathbf{k})$ reduced to the equation for $\tilde{\psi}(k)$ in the limit $p \rightarrow 0$. These possibilities result in the same series expansions for the thermodynamic quantities (47), (51) and (52) but produce different data for the long-range spatial boson correlations. Here we limited ourselves to considering the most simple variant of generalizing Eq. (12), which makes it possible to investigate only the thermodynamics of a strongly interacting Bose gas. The interesting and important problem of the spectrum of the elementary excitations is thus beyond the scope of this paper and will be the subject of future investigations.

\section{CONCLUSION}

Concluding, let us take notice of the important points of this paper once more. It was demonstrated that thermodynamically consistent calculations based on Eqs. (11) and (12) conclusively result in Eqs. (20)(22). Therefore, using the Bogoliubov model with the "dressed" interaction does not provide the satisfactory solution of the problem of the strong-coupling Bose gas. As it was shown, when investigating this subject, one should go beyond the Bogoliubov scheme. To do this, we developed the approach reduced to the system of Eqs. (33), (39), (40) and (41). These equations leading to the in-medium Lippmann-Schwinger equation (42), reproduce the familiar results $(47),(50)$ and (52) for the condensate depletion, chemical potential and mean energy but yield a completely different picture of the spatial boson correlations. This difference should manifest itself in the next orders of the density series expansions 
for the thermodynamic quantities and in the excitation spectrum as well.
This work was supported by the RFBR Grant No. 9702-16705.
[1] N. N. Bogoliubov, J. Phys. USSR 11, 23 (1947); reprinted in D. Pines, The many-body problem (W. A. Benjamin, New York, 1961).

[2] T. D. Lee, K. Huang, C. N. Yang, Phys. Rev. 106, 1135 (1957); K. A. Brueckner, K. Sawada, Phys. Rev. 106, 1117 (1957); S. T. Belyaev, Zh. Exp. Teor. Fiz. 34, 433 (1958) [Sov. Phys. JETP 7, 299 (1958)]; N. M. Hugenholtz, D. Pines, Phys. Rev. 116, 489 (1959).

[3] N. N. Bogoliubov, Lectures on quantum statistics. Vol. 1 (Gordon and Breach, New York, 1967), p. 39.

[4] A. Yu. Cherny, Pair wave functions in a Bose liquid, Eprint cond-mat/9807120.

[5] A. Yu. Cherny, A. A. Shanenko, Phys. Lett. A 250, 170 (1998).

[6] By the leading order we mean zero depletion of the condensate: $n=n_{0}$.

[7] A. A. Shanenko, Phys. Lett. A 227, 367 (1997); Phys.
Lett. A 231, 414 (1997).

[8] A. L. Fetter, J. D. Walecka, Quantum theory of manyparticle systems (McGraw-Hill, New York, 1971), p. 150.

[9] From the relation $\lim _{\mathbf{p} \rightarrow 0} \psi_{\mathbf{p}}(\mathbf{r})=\sqrt{2} \psi(r)$ an interesting limit can be derived with the help of Eqs. (5) and (6): $\lim _{\mathrm{p} \rightarrow 0} \lim _{V \rightarrow \infty} \sqrt{V}\left\langle a_{2 \mathrm{p}}^{\dagger} a_{\mathrm{p}+\mathrm{k}} a_{\mathrm{p}-\mathrm{k}}\right\rangle / n_{2 p}=$ $2\left\langle a_{\mathrm{k}} a_{-\mathrm{k}}\right\rangle / \sqrt{n_{0}}$.

[10] N. N. Bogoliubov, Quasi-averages, preprint D-781, JINR, Dubna (1961) [English transl. N. N. Bogoliubov, Lectures on quantum statistics. Vol. 2 (Gordon and Breach, New York, 1970), p. 1].

[11] It is not true in the case of complex-value wave functions. However, in this situation integrands in Eqs. (33) and (39) would include the absolute values of $\widetilde{\psi}(k)$ and $\widetilde{\psi}_{\mathbf{p}}(\mathbf{k})$ for which we have $\left|\widetilde{\psi}_{\mathbf{p}}(\mathbf{k})\right|-\sqrt{2}|\widetilde{\psi}(k)| \propto p^{2}$ at small $p$ in the case of the boundary conditions (4).

[12] R. P. Feynman, Phys. Rev. 91, 1291 (1953).

\title{
ДО УЗАГАЛЬНЕННЯ МОДЕЛІ БОГОЛЮБОВА НА ВИПАДОК СИЛЬНОГО ЗВ’ЯЗКУ
}

\author{
А. Ю. Чорний ${ }^{1}$, А. А. Шаненко ${ }^{2}$ \\ 1 Лабораторія нейтронной фізики ім. Франка, Об'єднаний інститут ядерних досліджень \\ 141980, Дубна, Московсъка обл, Росія \\ 2 Лабораторія теоретичной фізики ім. Боголобова, Об'єднаний інститут ядерних дослідюень \\ 141980, Дубна, Московсъка обл., Росіл
}

\begin{abstract}
Відомі результати про розріджений бозе-газ з короткосяжною відштовхувальною взаємодією вимагають перегляду через термодинамічну непослідовність методу, який значною мірою складає основу сьогоднішнього розуміння цьго явища, а також через неадекватну поведінку парної функції розподілу на малих міжбозонних віддалях. Стаття подає новий погляд на розріджений бозе-газ зі взаємодією довільної сили, використовуючи зведену матрицо густини другого порядку та варіящійну процедуру. Такий підхід дозволяє уникнути згаданої непослідовности та оперувати синпулярними потенпіялами, такими, як потенпіял Леннарда-Джонса. Увесь розгляд стосується нульової температури.
\end{abstract}

